\title{
Strukturwandel der Öffentlichkeit aus der Perspektive einer Theorie der Spätmoderne
}

\author{
Christian Strippel
}

\section{$1 \quad$ Einleitung}

Das Nachdenken über Strukturveränderungen von Öffentlichkeit ist in der Kommunikationswissenschaft wie der Soziologie üblicherweise stark an Theorien moderner Gesellschaften geknüpft. Der von Habermas (1962) beschriebene Strukturwandel hin zu einer bürgerlichen Öffentlichkeit steht zum Beispiel in der Tradition der Kritischen Theorie einer bürgerlich-kapitalistischen Gesellschaft; und systemtheoretische Konzeptionen etwa von Publizistik (Marcinkowski 1993) und Öffentlichkeit (Kohring 2006) sind in der Theorie funktional-differenzierter Gesellschaften in der Tradition von Parsons (1971) und Luhmann (1984) verankert. Kurt Imhof zufolge ist eine solche gesellschaftstheoretische Fundierung von Öffentlichkeitstheorie auch geboten, da Gesellschaft jenen normativen und strukturellen Referenzrahmen liefere, auf den bezogen sich Öffentlichkeit entfalte (Imhof 2003a). Umgekehrt seien Gesellschaft und ihr Wandel nur anhand von Öffentlichkeit und öffentlicher Kommunikation beobachtbar (Imhof 2003b). Öffentlichkeitstheoretische Fragestellungen und Probleme waren für ihn deshalb immer eng mit Theorien moderner Gesellschaften verknüpft (Imhof 2008).

Auch die Diskussion um einen erneuten Strukturwandel von Öffentlichkeit im Zeichen der Digitalisierung weist häufig gesellschaftstheoretische Bezüge auf. Die zentrale Rolle insbesondere des Internets bei der Eröffnung und Ausweitung politischer Partizipationsmöglichkeiten wurde etwa im Rahmen von Theorien zur „Netzwerkgesellschaft“ (Castells 2001) und „Netzwerköffentlichkeit“" (Neuberger 2014) ausgearbeitet; die „Plattformisierung“ öffentlicher Kommunikation (Eisenegger 2017) wurde in Arbeiten zur „Platform Society“ (van Dijck et al. 2018) und zum „Platform Capitalism“ (Srnicek 2016) behandelt; und auch die Transformationsprozesse der Digitalisierung, Datafizierung und Algorithmisierung wurden aus systemtheoretischer Perspektive bereits erörtert (Baecker 2007, 2018).

Dieser Beitrag möchte die gesellschaftstheoretisch fundierte Diskussion des neuen Strukturwandels der Öffentlichkeit fortführen und dabei einen neuen Ansatz aufgreifen, der von dem Kultursoziologen Andreas Reckwitz (2017a) unter dem Titel „Die Gesellschaft der Singularitäten - Zum Strukturwandel der Moderne“ 
vorgelegt wurde. Der Autor verfolgt in dieser Arbeit die These, dass sich in den Gesellschaften Europas und Nordamerikas ein Strukturwandel vollzogen habe, in dessen Zuge diese von einer sozialen Logik des Allgemeinen auf eine soziale Logik des Besonderen umgestellt hätten (S. 10-13). Während in der industriellen Moderne seit dem späten 18. Jahrhundert die formale Rationalisierung - im Sinne technischer Standardisierung, wissenschaftlicher Generalisierung und rechtlicher Formalisierung - dominiert habe (S. 28-46), zeichne sich die spätmoderne Gesellschaft seit den 1970er Jahren durch eine Kulturalisierung des Sozialen aus - die maßgebliche Ausrichtung und Bewertung aller sozialer Einheiten (Objekte, Subjekte, Kollektive, Räume und Zeiten) am Ideal des Singulären, also des kulturell Besonderen und Einzigartigen (S. 47-92). ${ }^{1}$

Im Folgenden wird dieser Theorieentwurf vorgestellt, diskutiert und auf seine Potenziale für eine Theorie von Öffentlichkeit unter Bedingungen eines neuerlichen Strukturwandels hin geprüft. Im Fokus steht dabei die Frage, welche Einsichten durch die Unterscheidung der sozialen Logiken des Allgemeinen und Singulären (sowie die damit verbundene These ihrer Umstellung) gewonnen werden können - und wie sie sich mit derzeitigen Überlegungen und Diskussionen in der Öffentlichkeitstheorie verbinden lassen. Im Verlauf dieses Beitrags wird darüber hinaus ein Vorschlag unterbreitet, wie der theoretischen Herausforderung begegnet werden kann, derzeitige Strukturveränderungen von Gesellschaft und Öffentlichkeit aus einer fachlichen Tradition heraus angemessen zu beobachten und zu beschreiben, die sich begrifflich und theoretisch in der industriellen Moderne entwickelt hat. Der vorliegende Text begreift sich dabei bereits als Versuch, dieser Herausforderung zu begegnen. ${ }^{2}$

\section{Die Gesellschaft der Singularitäten}

Was ist nun also damit gemeint, wenn es heißt, spätmoderne Gesellschaften hätten von einer sozialen Logik des Allgemeinen auf eine des Besonderen bzw. Singulären umgestellt? Um diese Frage zu beantworten, muss zunächst auf den sozialtheoretischen Hintergrund verwiesen werden, auf dessen Grundlage diese These entwickelt wurde. So argumentiert Reckwitz in seiner Arbeit nämlich durchgängig aus einer Perspektive der Praxistheorie in der Tradition von Bourdieu (1972), Giddens (1984) und Schatzki (1996), zu der er in der Vergangenheit auch selbst schon grundlegende Arbeiten vorgelegt hat (siehe etwa Reckwitz 2003). Das Verhältnis

Im Folgenden beziehen sich alle nicht näher gekennzeichneten Seitenangaben auf den hier im Fokus stehenden Titel „Die Gesellschaft der Singularitäten“ von Andreas Reckwitz (2017a).

2 Bedanken möchte ich mich bei Alexandra Keiner, Daniela Ruß, Moritz Klenk und Mark Eisenegger für viele hilfreiche Anmerkungen zu früheren Versionen dieses Beitrags. 
von individuellem Handeln und gesellschaftlicher Struktur wird in dieser Theoriefamilie im Anschluss an Giddens (1984) strukturationstheoretisch gefasst. Das Soziale wird demnach weder vom Akteur, noch von einer Struktur her beschrieben und erklärt, sondern beide treffen - so die Theorie - in sozialen Praktiken aufeinander und gehen in ihnen auf. Soziale Praktiken seien dabei situativ spezifische Verhaltensroutinen (,nexus of doings and sayings“, so Schatzki 1996: 89), die von einem Subjekt erlernt und (körperlich) eingeübt werden müssen. Dazu werden gesellschaftliche, also „kollektiv geteilte Wissensordnungen, Symbolsysteme, kulturelle Codes, Sinnhorizonte“ in „praktisches Wissen“ übertragen, „,inkorporiert" und habitualisiert sowie schließlich durch den Vollzug der Praktiken reproduziert (für alle Zitate siehe Reckwitz 2003: S. 286-297). Damit sei das Verhältnis zwischen sozialen Praktiken und gesellschaftlicher Ordnung rekursiv.

Das Erlernen und der Vollzug sozialer Praktiken würden sich dabei, so Reckwitz (2017a), an der sozialen Logik einer Gesellschaft ausrichten. Verstanden wird darunter eine „,umfassende Strukturierungsform“, die alle „Praktiken der Beobachtung, der Bewertung, der Hervorbringung und der Aneignung umfasst und zugleich alle Einheiten des Sozialen einschließt" (S. 37). Durch das rekursive Verhältnis zwischen sozialen Praktiken und gesellschaftlicher Ordnung richten sich allerdings nicht nur Personen in ihren Verhaltensweisen an der sozialen Logik einer Gesellschaft aus, sondern stellen diese dadurch umgekehrt auch selbst erst performativ her. Aus diesem Grund sei die soziale Logik einer Gesellschaft „,nicht kurzerhand objektiv oder subjektiv vorhanden, sondern durch und durch sozial fabriziert" (S. 13) - sowie weitestgehend implizit und unbestimmt. Sie könne also nicht einfach durch Wiederholung von Praktiken reproduziert, sondern müsse kontextspezifisch gedeutet werden, weshalb sowohl soziale Praktiken als auch die gesellschaftliche Ordnung beide interpretativ offen, variabel und immer veränderlich seien (Reckwitz 2003: S. 294).

Vor diesem Hintergrund behauptet Reckwitz (2017a) nun mit seiner Theorie, die industrielle Moderne sei bislang von einer sozialen Logik des Allgemeinen und von Praktiken der Allgemeinisierung (doing generality) geprägt gewesen. Solche Praktiken würden sich dadurch auszeichnen, dass sie „die soziale Welt rationalisieren“ und versuchen, ,ihr allgemeine Formen aufzupressen und sie in die Richtung allgemeiner Formen zu gestalten" (S. 29). Dies äußere sich etwa in einer allgemeinen Tendenz zur Typisierung und formalen Rationalisierung der sozialen Welt insbesondere durch Technik, Wissenschaft und Recht (S. 31-36). Dem gegenüber stellt Reckwitz die soziale Logik des Singulären, auf die insbesondere die Gesellschaften westlicher Industriestaaten seit den 1970er Jahren umgestellt hätten. Das Singuläre zeichne sich hier durch seine „Nichtverallgemeinerbarkeit, Nichtaustauschbarkeit und Nichtvergleichbarkeit" (S. 51) aus, weshalb die Praktiken der Singularisierung bzw. Besonderung (doing singularity) folglich in erster 
Linie auf die Produktion und Aneignung solcher unvergleichlicher Einzigartigkeiten abzielen würden (S. 64-71).

Die maßgebliche soziale Praktik der spätmodernen Gesellschaft ist für Reckwitz die Valorisierung - die Bewertung sozialer Einheiten als kulturell besonders, einzigartig und deshalb wertvoll. Eine solche Wertzuschreibung könne allerdings nicht beliebig vorgenommen werden, sondern es hänge von anderen $a b$, ob etwas Singularitätsstatus erlange oder nicht: „Es bildet sich eine Öffentlichkeit von Zuschauern oder Betrachtern, die Aufmerksamkeit und Anerkennung gewähren oder entziehen" (S. 149). Die Entscheidung dieses Publikums, ob etwas besonders bzw. einzigartig ist (seien es nun Menschen, Dinge, Ereignisse, Orte oder Tätigkeiten), sei Reckwitz zufolge dabei „durch mehr oder minder intensive Formen der Affizierung (Freude, Spannung, Abenteuer, Genuss, Selbstbildung, moralische Befriedigung, Sicherheit) geprägt" (S. 150). Nur was andere affizieren könne, habe also Potenzial, singularisiert zu werden und so Aufmerksamkeit auf sich zu ziehen. Alles Normale und Profane gelte hingegen als uninteressant und wertlos.

Mit der Umstellung der sozialen Logik vom Allgemeinen (in der industriellen Moderne) auf das Besondere bzw. Singuläre (in der Spätmoderne) sollen sich die sozialen Praktiken also grundlegend neu ausgerichtet haben. Für Reckwitz heißt dies allerdings weder, dass es Praktiken der Besonderung nicht auch vor dieser Umstellung schon gegeben hat - so verweist er etwa auf die Individualität in der Romantik oder die moderne Kunst (S. 96-100); noch solle damit gesagt sein, dass Praktiken der Allgemeinisierung in einer Gesellschaft der Singularitäten keinen Platz mehr hätten. Statt einer Ablösung der einen durch die andere Logik habe sich vielmehr ,das Verhältnis zwischen den sozialen Logiken des Allgemeinen und des Besonderen in den letzten 40 Jahren verändert [...]. Während die beiden Logiken in der industriellen Moderne einen asymmetrischen Dualismus bilden, transformieren sie sich in der Spätmoderne in einer Vordergrund- und Hintergrundstruktur" (S. 19). Die sozialen Praktiken der Allgemeinisierung hätten sich demnach so umgestellt, „dass sie ,im Hintergrund“ die Form von allgemeinen Infrastrukturen für die systematische Verfertigung von Besonderheiten annehmen“" (ebd.).

Bleibt noch zu klären, wie es zu dieser Umstellung der sozialen Logiken überhaupt kam. Reckwitz verortet diesen Übergang von der industriellen zur Spätmoderne in den letzten 20 bis 30 Jahren des vergangenen Jahrhunderts. In jener Zeit sei es zu einer "historischen Koinzidenz“ (S. 103) von drei sich einander verstärkenden Faktoren gekommen, die er als Treiber einer allmählichen gesellschaftlichen Transformation sieht: Zum ersten habe sich die industrielle Ökonomie nach dem Vorbild der ,creative economy“ und speziell des Kunstmarktes zu einer postindustriellen, kulturkapitalistischen Ökonomie entwickelt, in der vor allem kulturelle Güter - Dinge, Dienste, Ereignisse und auch Medienformate - auf hochkompetitiven Märkten um Aufmerksamkeit konkurrieren (S. 111-179). Dabei würden 
kreative Arbeiten in der Wissens- und Kulturökonomie (Kunst, Wissenschaft, Medien, Musik) aufgewertet, während routinisierte Arbeiten wie einfache Dienstleistungen (Kurierdienste, Logistik, Reparaturservice), Verwaltungs- und Infrastrukturarbeiten (Bürokratie, Müllentsorgung, Abwassertechnik) als profane „Normalisierungsarbeit“" (S. 184) abgewertet würden. Dies führe nicht nur zu einer Polarisierung zwischen den Berufsgruppen, sondern auch innerhalb der Wissens- und Kulturökonomie, in der es durch das, Winner-take-all'-Prinzip zu Spannungen zwischen einigen wenigen „Superstars“ und den prekären Arbeitsbedingungen der meisten anderen komme (S. 186-223).

Als zweiten Faktor für den gesellschaftlichen Strukturwandel sieht Reckwitz die digitale Technologie mit dem Internet als „Kulturmaschine“ (S. 227), die eine umfangreiche Kulturalisierung des Sozialen überhaupt erst möglich gemacht hätten (S. 225-229). Auf Grundlage der Errungenschaften technischer Standardisierung - Digitalisierung, Computing und Vernetzung - stellen sie eine globale Plattform für die massive, radikal demokratisierte Produktion von Kulturformaten bereit, auf der ein chronischer Bedarf an Aufmerksamkeit und ein entsprechend starker, affektbasierter Sichtbarkeitswettbewerb herrsche (S. 229-243). Gleichzeitig würden die Nutzerinnen und Nutzer über datengetriebene, individualisierte Beobachtungsprofile durch die Technik selbst singularisiert und auf dieser Grundlage mit auf sie zugeschnittenen Internet-Umwelten versorgt (S. 243-265).

Reckwitz identifiziert in diesem Zusammenhang fünf ,Spannungsfelder der Netzkultur“ (S. 265): Erstens bestehe eine „,soziale Erwartung, einzigartig zu sein, und zwar auf akzeptable Weise“" (S. 266): Weder sollte man sich zu konformistisch geben, noch dürfe die eigene Individualität negativ konnotiert sein - etwa durch beschämende Peinlichkeiten oder pathologisierbare Zuschreibungen wie Kriminalität, Extremismus, Gewalttätigkeit, Sucht oder sexuelle Perversion (S. 226-267). Zweitens würden personalisierte Filterblasen, digitale Neogemeinschaften und das „strukturelle Nichtvergessen des Netzes“ (S. 268) das Experimentieren mit und das Wechseln zwischen verschiedenen Identitäten erschweren und so einmal gewählte Identitäten stabilisieren. Dies führe drittens zur „Erosion des Allgemeinen“ (S. 268) und einer Fragmentierung der Öffentlichkeit (siehe dazu Kapitel 3.1, unten). Viertens bilde sich ein „Regime des affektiven Aktualismus“ (S. 269) aus, in dem aktuelle und emotional aufgeladene Ereignisse starke mediale Beachtung fänden, während langfristigere Entwicklungen und eher neutrale Themen marginalisiert würden; und fünftens herrsche schließlich eine ,digitale Affektkultur der Extreme" (S. 270), die zwischen phatischer Wohlfühl-Kommunikation unter Gleichgesinnten und kommunikativer Aggressivität schwanke.

Als dritten Treiber nennt Reckwitz den sozio-kulturellen Wandel, den er als „Authentizitätsrevolution“ (S. 103) bezeichnet. Das romantische Ideal der Selbstverwirklichung habe sich mit der bürgerlichen Statusorientierung zur paradoxen 
Formel „erfolgreicher Selbstverwirklichung“ verbunden (S. 285-289) und drücke sich nun in einer ,Ethisierung“ (S. 292) und „Authentifizierung“ (S. 293) des Alltags und dem bedachten Kuratieren von Ernährung, Wohnen, Reisen, Körper, Bildung und Erziehung aus (S. 295-335). ${ }^{3}$ Eine solche „,singularistische Lebensführung" (S. 273) entfalte sich vor allem in der akademischen Mittelklasse, habe aber auch darüber hinaus Strahlkraft erlangt, sodass sie die kulturellen Maßstäbe auch für den Rest der Gesellschaft setze.

Von der akademischen Mittelklasse unterscheidet Reckwitz hier eine nichtakademische Mittelklasse und eine Unterklasse. Diese „Klassengesellschaft“ existiere ,jedoch nicht nur im engen materiellen Sinne, vielmehr handelt es sich auch und gerade um kulturelle Klassen: Neben den ungleich verteilten materiellen Ressourcen (Einkommen und Vermögen) unterscheiden sich diese Klassen hinsichtlich ihrer Lebensstile - und ihres kulturellen Kapitals“ (S. 275). Die Erosion der Industriegesellschaft und die Bildungsexpansion hätten zu einem „Paternostereffekt“ (S. 277; im Gegensatz zum „Fahrstuhleffekt“” bei Beck 1986: 121-130) des kulturellen Auf- und Abstiegs im Mittelstand geführt, der in eine stark polarisierte „Drei-Drittel-Gesellschaft“ (S. 282) gemündet sei (siehe dazu ausführlicher Reckwitz 2019: 63-133). Die superreiche Oberklasse bilde hingegen keine eigene kulturelle Klasse, da sie ein „,genuines Resultat des ,Winner-take-all'-Prinzips der Ökonomie der Singularitäten" sei und sich von der akademischen Mitteklasse deshalb ,weniger durch ihr kulturelles als vielmehr durch ihr ökonomisches (sowie soziales) Kapital“ (S. 281) unterscheide.

Aus dieser Konstellation ergeben sich Reckwitz zufolge ebenfalls zahlreiche Spannungsfelder nicht nur in der akademischen Mittelklasse mit ihrem singularistischen Lebensführung (Verzichtsaversion, Selbstzwang, Enttäuschungsanfälligkeit, S. 342-349), sondern auch und vor allem zwischen den Klassen. Es komme zu einer „Kulturalisierung der Ungleichheit“ (S. 350) in Form einer negativen Abgrenzung der akademischen Mittelklasse vom bürgerlichen Lebensstil der nichtakademischen Mittelklasse und dem „,muddling through“ (S. 350-355) der Unterklasse. Der damit verbundenen Entwertung, die bis zur Stigmatisierung insbesondere der Unterklasse reicht, wird innerhalb der abgewerteten Klassen wiederum durch die Kulturalisierung des eigenen Lebensstils begegnet - etwa durch die Hervorhebung von Lebenserfahrung, die Inszenierung klassischer Geschlechterrollen oder die Ablehnung von Elitismus, Kosmopolitismus, Multikulturalismus und Diversität als Symbole des singularistischen Lebensstils (S. 355-363).

Unter „Authentifizierung“" versteht Reckwitz die Entfaltung des „Selbst in seiner Besonderheit“ (S. 104). Das spätmoderne Leitmotiv des Strebens nach Authentizität führe dabei zwangsläufig zu der Paradoxie einer ,performativen Authentizität“ (S. 247), weshalb Authentizität immer als „Authentizitätsperformanz“ (S. 138) verstanden werden müsse. 


\subsection{Kritische Besprechung}

Von Reckwitz' Buch liegen inzwischen zahlreiche Besprechungen und Rezensionen aus diversen Disziplinen vor (Bach 2018, Frank 2018, Gümüsay 2018, Kilian 2018, Meyen 2018, Köhler 2019, Wagner, 2019). Auf dem soziologischen Nachrichtenportal Soziopolis wurde ein gesondertes Forum eingerichtet, in dem die Arbeit ausführlich diskutiert wurde (Knöbl 2017, Koppetsch 2017, Trautmann 2017, Vogel 2017, Saar 2018, Hohnsträter 2018, Rosa 2018, Moebius 2018, Reckwitz 2017b, 2018); und auf dem 39. Kongress der Deutschen Gesellschaft für Soziologie 2018 fand eine Podiumsdiskussion zu dem Buch statt, deren Beiträge inzwischen auch schriftlich vorliegen (Löw 2019, Reitz 2019, Schnabel 2019). Um nun zu prüfen, welche Einsichten die Theorie für die Diskussion eines digitalen Strukturwandels der Öffentlichkeit bereithält, wird im Folgenden die wichtigste Kritik an Reckwitz' Arbeit aus diesen Besprechungen zusammengetragen und anschließend hinsichtlich ihrer Bedeutung für die Theoriearbeit zum digitalen Strukturwandel der Öffentlichkeit diskutiert (siehe Kapitel 2.2).

Zunächst die Würdigungen: Bis auf wenige Ausnahmen finden sich in allen Besprechungen anerkennende Worte zu Reckwitz‘ Buch. Rosa (2018) zufolge besteche es dadurch, dass es dem Autor gelungen sei, ,die Moderne als soziale Formation im Ganzen zu denken und dabei zugleich ihre inneren Brüche und Widersprüche sichtbar werden zu lassen“. In einer „großartigen Verdichtungsleistung“, so findet auch Koppetsch (2017), habe Reckwitz „,ganz disparate Aspekte der Gegenwartsgesellschaft in ein gemeinsames Bild gebannt“. Laut Knöbl (2017) sei es ihm dabei gelungen, ,,den thematisierten empirischen Phänomenen immer wieder interessante und bis dato ungesehene Aspekte abzugewinnen"; Frank (2018) sieht die Stärke der Arbeit darin, ,das Augenscheinliche [...] nicht einfach anekdotisch aneinanderzureihen, sondern es theoretisch zu fassen “ (S. 73); Hohnsträter (2018) spricht von „feinen Beobachtungen“, die „einer Reihe von akademischen Fächern und öffentlichen Debatten Anregung und Anstoß zur Auseinandersetzung" liefern könnten; und Moebius (2018) zufolge gehört das Buch „unzweifelhaft zu den derzeit anregendsten Büchern im soziologischen Feld“".

Neben diesem Lob findet sich in den meisten Besprechungen aber auch Kritik an der Arbeit. Bemängelt werden etwa der Kulturbegriff (Knöbl 2017, Koppetsch 2017, Hohnsträter 2018) sowie die Unschärfe des Singularitätskonzepts aufgrund der fehlenden Unterscheidung des ,Einzigartigen “ vom ,Besonderen“ (Reitz 2019: 13). Auch die Abgrenzung von industrieller und Spätmoderne anhand ihrer sozialen Logiken wird als schwer haltbar kritisiert (Knöbl 2017, Koppetsch 2017, Köhler 2019: 111). In anderen Punkten gibt es hingegen widersprüchliche Positionen, etwa in der Einschätzung, ob Reckwitz' Klassentheorie „einem strukturalen, wenig dynamischen Bild der Klassengesellschaft verhaftet bleibt“ (Koppetsch 2017) 
oder sie im Gegenteil ein ,dynamisches Wechselspiel von Auf- und Abstieg“(Vogel 2017) beschreibt. Ebenfalls ist umstritten, ob die Theorie „,hartnäckig empirieresistent“ (Vogel 2017) sei oder ,ihre empirische Anwendung und Fundierung gleich mitliefert“ (Saar 2018). Schließlich wird bemerkt, dass Reckwitz „eine nicht unbekannte Geschichte“ (Reitz 2019: 12) erzählt, deren wichtigste Elemente „fast alle schon früher erörtert" (ebd.: 18) wurden. Verwiesen wird dabei etwa auf Simmel (1901), Eberlein (2000) oder Boltanski und Chiapello (2003).

Für das hier verfolgte Vorhaben einer öffentlichkeitstheoretischen Reflexion von Reckwitz“ Theorie ist jedoch eine andere Kritik relevanter: Sie betrifft erstens die erkenntnistheoretische Position der Theorie, zweitens ihre theoretische Tiefe und empirische Reichweite sowie drittens ihre Gültigkeit als Gesellschaftstheorie. Die Kritik an der erkenntnistheoretischen Position zielt dabei auf Reckwitz' Versuch ab, eine „kritische Analytik der Gegenwart“ (S. 23) vorzulegen, ohne normativ zu werden und einem kulturkritischen „Entlarvungsreduktionismus“ (S. 13) zu verfallen. Koppetsch (2017) bemängelt diesbezüglich, dass es ihm kaum gelungen sei, ,die Klassenspezifik seiner eigenen Relevanz- und Bewertungskriterien zu reflektieren“. Stattdessen verwechsele er , die Selbstbeschreibung der spätmodernen Gesellschaft mit ihren Strukturen“, die jedoch „,ihre Bedeutung im Normalfall gerade nicht ihrer Sichtbarkeit, sondern ihrer Unsichtbarkeit verdanken“. Mit der Zurückweisung jeglicher Normativität habe er sich nicht nur einen blinden Fleck für die eigene Position eingehandelt, sondern auch einen Mangel an Skepsis gegenüber den (von ihm) gemachten Beobachtungen von (für ihn) sichtbaren Phänomenen. Theoretisch bliebe daher ,völlig unklar, wie diese Struktur der Moderne aussieht“ (Rosa 2018); und empirisch wird ihm unterstellt, dass er ,seinem eigenen Milieu zu viel Bedeutung beimisst“" (Frank 2018: 73).

Gebündelt werden diese Kritikpunkte in der häufigen Bezeichnung der Arbeit als Zeit- oder Gesellschaftsdiagnose, mit der ihr (mal mehr, mal weniger explizit) der Status einer Gesellschaftstheorie abgesprochen wird. Dass mit diesem Etikett eine Abwertung verbunden ist, zeigt ein Blick in die soziologische Fachdiskussion zum Thema (siehe Bogner 2018: 9-26): So sieht Honneth (1994) Gesellschaftsdiagnosen als „Produkte einer Überverallgemeinerung von gesellschaftlichen Entwicklungen“( (S. 8), denen man „ein erhebliches Maß an Skepsis“ (S. 9) entgegenbringen müsse; laut Reese-Schäfer (1996) gelten sie ,als interessant, aber doch auch als ein wenig unsolide“" (S. 377); und Kieserling (2002) bescheinigt der Soziologie, selbst eine „Diagnosegesellschaft“ zu sein, die sich dadurch auszeichne, „sich unter dem Beistand ihrer Massenmedien alle paar Jahre einzureden, an einer Schwelle zu einer ganz neuen Epoche zu stehen" (S. 68). Diese Kritik griff Osrecki (2011) später auf und machte die mutmaßliche Ausrichtung von Gesellschaftsdiagnosen an massenmedialer Aufmerksamkeit zum zentralen Unterscheidungskriterium gegenüber Gesellschaftstheorien. 


\subsection{Gesellschaftsdiagnosen als gesellschaftstheoretische Exploration}

Die Frage, ob es sich bei Reckwitz‘ Arbeit nun um eine Gesellschaftstheorie oder eine Gesellschaftsdiagnose handelt, ist für das Vorhaben einer gesellschaftstheoretischen Information der Theoriearbeit zum Strukturwandel von Öffentlichkeit selbstverständlich nicht unerheblich; gleichwohl steht sie einem solchen Vorhaben aber auch nicht im Weg. Ganz im Gegenteil wird hier die These vertreten, dass gerade soziologische Gesellschaftsdiagnosen gewinnbringend für die Theoriearbeit zu gesellschaftlichen Strukturveränderungen herangezogen werden können, reagieren sie doch auf die erkenntnistheoretische Herausforderung, vor der Soziologie und Kommunikationswissenschaft stehen, sobald sie versuchen, mit ihren an der Industriegesellschaft geschulten Begriffen und Theorien jene sich wandelnden Gesellschaftsstrukturen zu beschreiben, die sich - so ja immerhin die These des Strukturwandels - von eben dieser Industriegesellschaft radikal unterscheiden (siehe auch Reckwitz 2017a: 429, 2017b). ${ }^{4}$ Ein solches Vorhaben kann, so meint Beck (1986), „nur in einem harten Ringen gegen die alten mit ,post' über sich selbst verlängerten Theorien und Denkgewohnheiten gelingen" (S. 12).

Vor diesem Hintergrund können Gesellschaftsdiagnosen gerade durch ihre vielfach kritisierten Mittel der Vereinseitigung und Spekulation interessant werden. Zum einen können durch die Fokussierung auf einzelne Aspekte des Strukturwandels (im Sinne einer Komplexitätsreduktion) zunächst im Kleinen neue Perspektiven auf Gesellschaft und Öffentlichkeit erarbeitet werden, die sich dann für die weitere soziologische Gegenwartsbeschreibung nutzen ließen. Junge (2016) spricht deshalb auch von einer „Fokussierungsfunktion“ (S. 51) von Gesellschaftsdiagnosen für die Soziologie. Zum anderen liegt die Stärke von Gesellschaftsdiagnosen gerade darin, dass sie, wie Beck (1986) schreibt, nicht ", die noch vorherrschende Vergangenheit", sondern „die sich heute schon abzeichnende Zukunft" (S. 12-13) in den Blick nehmen. Unter der Bedingung des herrschenden Zeitverständnisses, demzufolge „die Gegenwart aus der Unterscheidung von Vergangenheit und Zukunft definiert wird“ und damit „das in die Zeit eingeschlossene ausgeschlossene Dritte, weder Zukunft noch Vergangenheit, aber zugleich auch das eine und das andere“ (Luhmann 1987: 167) ist, geben Gesellschaftsdiagnosen also „der Zukunft einen Primat über die Bestimmung der Gegenwart“ (ebd.). Wenn Gegenwart aber als ,zukünftige Vergangenheit“ verstanden wird, muss die Gegenwartsgesellschaft als das beschrieben werden, was sie noch nicht ist. Im Gegensatz zu

Dies gilt umso mehr, als sowohl, Gesellschaft" und ,Moderne' als auch die Soziologie und Kommunikationswissenschaft, die um deren Beobachtung und Beschreibung bemüht sind, selbst, Erfindungen " moderner Gesellschaften sind. Lichtblau bezeichnet Zeitdiagnosen deshalb auch als „Moderne im Selbstbezug“ (1991) und „Selbstunterscheidungen der Moderne“ (1998). 
deduktiver Theorieanwendung und empirisch getriebener Theorieentwicklung haben wir es bei Gesellschaftsdiagnosen also mit „projektiver Gesellschaftstheorie“ (Beck 1986: 13) zu tun.

Aufgrund dieser Potenziale für die Theoriearbeit zu Strukturveränderungen in Gesellschaft und Öffentlichkeit wird hier also dafür plädiert, Gesellschaftsdiagnosen nicht als „,medialisierte Intellektuellendiskurse“ (Osrecki 2011: 317) aufzufassen und dadurch theoretisch abzuwerten, sondern sie als gesellschaftstheoretische Exploration im Sinne eines induktiven Verfahrens der Theoriearbeit zu verstehen und dementsprechend ernst zu nehmen. Damit wird die Unterscheidung zwischen Gesellschaftstheorien und -diagnosen nicht mehr wissenssoziologisch über das vermeintliche Publikum (Wissenschaft vs. Medien) definiert, sondern methodisch über ihren Zugriff auf eine sich wandelnde Gesellschaftsstruktur durch Fokussierung auf (einzelne) Strukturveränderungen und spekulativ verallgemeinernde Projektion. Damit sei selbstverständlich nicht gesagt, das analytische Potential von Gesellschaftstheorien solle für Theoriearbeit zu Strukturveränderungen von Gesellschaft und Öffentlichkeit gänzlich außer Acht gelassen werden; es gilt jedoch zu hinterfragen, inwieweit die Fortführung tradierter Perspektiven auch blind machen kann für genau das, was es zu beobachten gilt.

\section{Die Öffentlichkeit einer Gesellschaft der Singularitäten}

Welche öffentlichkeitstheoretisch relevanten Aspekte bekommt man nun also in den Blick, folgt man Reckwitz' Theorie spätmoderner Gesellschaften der Singularitäten? Im Folgenden werden auf diese Frage beispielhaft drei Antworten gegeben: Zum ersten wird gezeigt, welche Rolle Öffentlichkeit für eine solche Gesellschaft aus der Sicht dieser Theorie überhaupt (noch) spielen könnte. Es folgt dann zweitens eine Auseinandersetzung mit möglichen Folgen, die sich aus einer Umstellung der sozialen Logik vom Allgemeinen auf das Besondere für den Journalismus als eines der zentralen Berufsfelder des Arbeitens an Öffentlichkeit ergeben. Und drittens wird schließlich darauf eingegangen, wie sich vor diesem Hintergrund auch die öffentliche Kommunikation verändert.

\section{1 Öfentlichkeit}

Die Frage nach der Rolle von Öffentlichkeit für eine Gesellschaft der Singularitäten streift Reckwitz in seiner Arbeit nur beiläufig. Explizit geht er darauf erst am Ende des Buches ein, als er eine „Krise des Allgemeinen“ diagnostiziert, „in die eine Gesellschaft gerät, die sich radikal am Besonderen ausrichtet" (S. 435). Besonders zum Ausdruck komme diese Krise ihm zufolge im Bereich des Politischen 
und - damit eng verbunden - der Öffentlichkeit: „Die Krise des Politischen“, so schreibt er, „betrifft sowohl die politische Öffentlichkeit als auch den Staat. Nicht zuletzt angetrieben durch die Entwicklung der digitalen Medien, verlagert sich die politische Debatte in autonome Teilöffentlichkeiten“ (S. 434), was zu einer „Parzellierung der politischen Öffentlichkeit in diverse Communities" (S. 436) und so zum Verlust ihrer gesellschaftlichen Integrationskraft führe. Obwohl hier nur von der politischen Öffentlichkeit die Rede ist, weitet Reckwitz seinen Befund schließlich zu einer allgemeinen „Krise der Öffentlichkeit“ (ebd.) aus.

Über die Problematik autonomer Teilöffentlichkeiten und eine Krise der Öffentlichkeit ist auch bei Imhof (2011) schon zu lesen. Insofern handelt es sich hierbei mitnichten um eine Erkenntnis, zu der sich erst mit Hilfe von Reckwitz' Theorie kommen ließe. Tatsächlich ist Imhofs Krisenanalyse sogar weitaus differenzierter angelegt, dekliniert er seinen Befund doch ausführlich anhand der Deliberations-, Legitimations- und Integrationsfunktion von Öffentlichkeit für moderne Gesellschaften durch (S. 108-148). Mit seiner Theorie, die vor allem die Entgrenzung von Ökonomie, die Schwächung des Nationalstaats, eine kulturelle Segregation sowie die Ausdifferenzierung des Mediensystems für die von ihm diagnostizierte Krise der Öffentlichkeit verantwortlich macht, ließe sich also weitaus mehr sehen als mit Reckwitz' Problematisierung einer vermeintlichen Fragmentierung von Öffentlichkeit in „,singularisierte Umwelten“ (S. 259) und Filterblasen digitaler Neogemeinschaften und Communities (siehe dazu S. 258-265).

Um mit Reckwitz' Arbeit eine neue Perspektive auf Öffentlichkeit zu gewinnen, muss man sich stattdessen noch strenger an den zentralen Unterscheidungen und Argumentationslinien der Theorie orientieren als dies Reckwitz hier selbst tut. Denn angesichts dessen, dass er zu Beginn seiner Arbeit versichert, mit der Umstellung der sozialen Logik vom Allgemeinen auf das Besondere gehe keinesfalls ein Verschwinden des Allgemeinen einher, sondern vielmehr ein Spannungsverhältnis von „Vordergrund- und Hintergrundstruktur“ (S. 19), kommt der Befund einer „Krise des Allgemeinen“ doch überraschend. Zwar versucht Reckwitz, diesen Widerspruch aufzulösen, indem er betont, von dieser Krise des Allgemeinen sei die Hintergrundstruktur der formalen Rationalisierung gar nicht betroffen, sondern nur „das sozial, kulturell und politisch gemeinsam Geteilte“, wie etwa „gemeinsame, reziproke Anerkennungsformen, gemeinsame Systeme des kulturell Wertvollen sowie gesamtgesellschaftliche Kommunikationsformen und normative Rahmungen“" (S. 437). Doch kann auch dies nicht überzeugen, da die Infragestellung des ,gemeinsam Geteilten“ auch der industriellen Moderne nicht fremd gewesen sein dürfte (siehe auch Trautmann 2017) und darüber hinaus nicht einleuchtet, warum die Ausrichtung einer Gesellschaft an der Logik des Besonderen zu einer Krise des Allgemeinen auf der inhaltlichen Ebene dieses ,gemeinsam Geteilten“, nicht aber auf der Ebene der Infrastruktur führen soll. 
Deshalb gilt es hier Reckwitz' Ausgangsthese, mit der Umstellung der sozialen Logik habe sich lediglich das Verhältnis zwischen dem Allgemeinen (als ermöglichende Infrastruktur im Hintergrund) und dem Besonderen bzw. Singulären (an der sichtbaren Oberfläche im Vordergrund) verändert, noch einmal stärker zu machen. Mit Blick auf einen möglichen Strukturwandel der Öffentlichkeit ließe sich dann schlussfolgern, dass Öffentlichkeit keineswegs in eine Krise gekommen sei - dieser Befund ist für eine auf maximale Aufmerksamkeit abzielende Gesellschaft der Singularitäten auch nicht plausibel -, sondern stattdessen zwei Strukturebenen von Öffentlichkeit zu unterscheiden sind, deren Verhältnis sich im Zuge der Umstellung der sozialen Logik grundlegend verändert hat.

Zum einen hätten wir es demnach mit einer Hintergrund- bzw. Infrastruktur von Öffentlichkeit zu tun, in der die sozialen Praktiken öffentlicher Kommunikation dem Imperativ des doing generality folgen. Es liegt nahe, hier zunächst an die technische Infrastruktur zu denken, die etwa durch Intermediäre wie Suchmaschinen, Nachrichtenaggregatoren, soziale Netzwerke und Videoplattformen bereitgestellt wird, um öffentliche Kommunikation (im Internet) durchsuchbar, übersichtlich und anschlussfähig zu machen. Durch die ihnen jeweils eigene (zumeist algorithmische) Organisation von Inhalten und Publika prägen sie die Infrastruktur von Öffentlichkeit maßgeblich mit (siehe Schmidt et al. 2017, Stark/Magin 2019). Darüber hinaus gilt es, hier aber auch an eine journalistische Infrastruktur zu denken, die sich durch die „Herstellung und Bereitstellung von Themen zur öffentlichen Kommunikation" (Rühl 1980: 322-323) auszeichnet. Durch die institutionalisierte Ausrichtung an professionellen Routinen, Standards und Normen wird Öffentlichkeit thematisch nach Relevanz strukturiert, zuverlässig auf Dauer gestellt und dadurch überhaupt erst erwartbar und inhaltlich anschlussfähig gemacht (siehe Jarren 2008, 2015). Zusammen mit den technischen Intermediären und nicht zuletzt auch den Massenmedien stellt der Journalismus also jene Infrastruktur bereit, ohne die eine Gesellschaft nicht wüsste, worüber sie zu reden habe.

Auf Grundlage dieser Infrastruktur kommt es dann mit Reckwitz (2017a) zu einer ,systematische[n] Verfertigung von Besonderheiten“ (S. 19) in der Vordergrund- bzw. Suprastruktur von Öffentlichkeit, in der sich die Praktiken öffentlicher Kommunikation an dem Imperativ des doing singularity ausrichten. In affizierender Form werden hier zum einen diejenigen Informationen und Themen, die vom professionellen Journalismus zur öffentlichen Kommunikation bereitgestellt werden, (positiv oder negativ) bewertet, ggf. entsprechend kommentiert und weiterverbreitet; zum anderen bemühen sich hier Einzelpersonen, Gruppen, Organisationen und auch politische und staatliche Akteure - unter Rückgriff auf die technische Infrastruktur - um möglichst viel Aufmerksamkeit für ihre jeweiligen Anliegen (siehe Hasebrink 2019: 411-412). Angesichts seiner großen Reichweite und der damit verbundenen Aussicht auf maximale Aufmerksamkeit ist insbesondere 
der massenmediale Journalismus dabei die ultimative Adresse solcher Bemühungen. Die öffentliche Suprastruktur wird damit geprägt durch Anschlusskommunikation an journalistische Inhalte (siehe Nuernbergk 2013) sowie um Aufmerksamkeit und positive Valorisierung bemühte öffentliche Kommunikation andererseits. So entstehen ,affective publics“ (Papacharissi 2015), „dissonante Öffentlichkeiten“ (Pfetsch/Löblich/Eilders 2018) und eventuell auch jene Partikularöffentlichkeiten der Neogemeinschaften, die Reckwitz vorrangig im Blick hat.

Die Modellierung von Öffentlichkeit als Doppelstruktur ergänzt bestehende Öffentlichkeitsmodelle wie das zweiseitige Arenamodell (Akteursarena vs. Publikumsgalerie; Gerhards/Neidhardt 1990), das vertikale Ebenenmodell (Donges/Jarren 1998) oder das Zentrum-Peripherie-Modell der Gegenöffentlichkeiten (Fraser 1990) um eine neue Dimension der zweifachen Strukturhaftigkeit, in sich öffentliche Kommunikation an unterschiedlichen Logiken bzw. Imperativen ausrichtet. Die Doppelstruktur kann dabei insofern als neuartig angesehen werden, als öffentliche Kommunikation bislang nur im journalistisch-massenmedialen Rahmen möglich war und sich deshalb an der Medienlogik orientieren und Praktiken der Allgemeinisierung unterordnen musste. Mit dem Aufkommen des Internets vollzog sich dann auch im Bereich der öffentlichen Kommunikation ein „Aufstand des Publikums“ (Gerhards 2001), in dem sich „das ,Publikum an sich“ in ein ,Publikum für sich““(S. 180) transformierte und höhere Inklusionsansprüche einforderte bzw. sie unter Rückgriff auf die neu geschaffene technische Infrastruktur einfach (selbst) realisierte. Dadurch entstand eine Vielfalt neuer Formen öffentlicher Kommunikation, die zwar - wie oben beschrieben - an die journalistische Infrastruktur anschließen, dabei jedoch eine eigene Struktur herausgebildet haben, die maßgeblich von Praktiken der Besonderung geprägt ist.

Doch auch umgekehrt bleibt die journalistische Infrastruktur von der sozialen Logik des Besonderen nicht unbeeinflusst. Aus dem kulturökonomischen Imperativ heraus, sich in einer Gesellschaft der Singularitäten als besonders bzw. einzigartig zu positionieren, kann sich der professionelle Journalismus nicht mehr erlauben, sich nicht an den Erwartungen seines Publikums zu orientieren (siehe Loosen/ Schmidt 2012, Haim 2019) und jene Themen als relevant aufzugreifen, die durch Affizierung für Aufmerksamkeit sorgen: Öffentlichkeitsresonanz wird zum Nachrichtenfaktor (Fürst 2013). Die journalistischen Infrastrukturpraktiken der Allgemeinisierung treten dadurch insofern in den Hintergrund, als sie hinter der , unterschiedslosen Masse“ an „Content" im Internet „unsichtbar“ werden (Neuberger 2002). Das bedeutet nicht, dass Journalismus nicht mehr also solcher erkennbar wäre (dies ist schließlich auch bei anderen Infrastrukturen wie Eisenbahnschienen oder Abwasserrohren nicht der Fall), angesichts der vielen aufmerksamkeitsstarken Alternativen - steile Thesen, spektakuläre Behauptungen, starke Meinungen und haarsträubende Geschichten - büßt er jedoch an Sichtbarkeit ein. Sie wird ihm 
nur noch dann explizit zuteil, wenn sein Funktionieren als Infrastruktur für Öffentlichkeit in Frage steht. So heißt es in der Infrastrukturforschung: „The normally invisible quality of working infrastructure becomes visible when it breaks" (Star 1999: 382). Die Diskussionen um die Gefährdung der Öffentlichkeit durch Desinformationen (siehe Schatto-Eckrodt et al. 2019) wären dafür ein Beispiel.

\subsection{Journalismus}

Angesichts der Bedeutung des Journalismus für die Infrastruktur für Öffentlichkeit hat dieser Berufszweig auch in der spätmodernen Gesellschaft der Singularitäten einen festen Platz. Verstärkt wird dies noch dadurch, dass es sich bei Medienberufen wie dem Journalismus auch in der industriellen Moderne bereits um kulturalisierte Tätigkeiten handelte („Kulturindustrie“, Horkheimer/Adorno 1944, siehe dazu Reckwitz 2017a: 100-102). Sie zählen für Reckwitz deshalb auch zur ,creative economy' (S. 115) und können sich damit von eher funktional-standardisierten Berufen kulturell abheben. Journalistische Praktiken lassen sich dabei gleich dreifach singularisieren: erstens als kreative Arbeit (S. 186-191); zweitens als Arbeit in bekannten und damit sichtbaren Organisationen (S. 191-200); und drittens als „Arbeit an Singularitäten“ (S. 182), also an Kultur- und Affektgütern, die sich selbst singularisieren lassen (dazu S. 119-126, 132-137) - etwa wenn über besondere Ereignisse oder Personen berichtet wird.

Für die journalistische Arbeit und ihre gesellschaftliche Wertschätzung gehen damit jedoch nicht nur Vorteile einher. So können die von Reckwitz (2017a) beschriebene „Polarisierung der Arbeitswelt“ (S. 183) und die „Kulturalisierung der Ungleichheit" (S. 350) in Folge der Auf- und Abwertung ganzer Berufsfelder und Lebensstile in spätmodernen Gesellschaften etwa zu einer Entfremdung zwischen Medienschaffenden, die sich überwiegend aus der akademischen Mittelklasse rekrutieren, und ihrem Publikum führen - und sich sogar bis zu antielitär gefärbter „Medienfeindlichkeit“ (Schindler et al. 2018) und Misstrauen gegenüber der Profession entwickeln (siehe Krüger 2016, Müller 2018).

Darüber hinaus gilt für den Journalismus auch, was Reckwitz über Spannungen innerhalb der Kreativbranche beschreibt: Denn auch hier lassen sich große Statusunterschiede und eine starke Prekarisierung der Arbeitsbedingungen vieler Journalistinnen und Journalisten beobachten. So sind es vor allem bestimmte Ressorts (z.B. Politik, Feuilleton, Sport), journalistische Formate (Reportage/Feature, Interview/Portrait, Kolumne/Leitartikel) und Praktiken (etwa Investigativrecherchen), die in der Lage sind, zu affizieren. Mit Reckwitz ließe sich hier von einem Journalismus des Besonderen sprechen, der innerhalb der Profession hoch angesehen ist und dementsprechend auch gut bezahlt wird. Demgegenüber steht ein Journalismus des Allgemeinen, der insbesondere die sachliche Berichterstattung 
sowie eher unsichtbare Arbeiten etwa in der Moderation von Nutzerkommentaren, dem Fact Checking oder im Lektorat umfasst (siehe dazu Blöbaum 2000). Angesichts der nahezu handwerklichen Routinisierung der hier angesiedelten Tätigkeiten kommt es in diesem Bereich zu einer starken Entwertung und einer entsprechende Prekarisierung der Arbeit (siehe etwa Kramp 2013).

Ein Beispiel für eine solche Entwertung liefert etwa die unter Begriffen wie „robot journalism“ (Clerwall 2014), ,automated journalism“ (Graefe 2016) und „algorithmic journalism“ (Dörr 2016) geführte Diskussion um verschiedene Einsatzmöglichkeiten von „,maschinellem Lernen“ im Journalismus. Die Spekulation darüber, ob sich etwa Meldungen automatisiert schreiben oder Nutzerkommentare durch Algorithmen moderieren ließen, geht insofern mit einer Abwertung der entsprechenden Arbeiten einher, da ihnen dadurch eine leicht zu replizierende Profanität unterstellt wird. Dass allein solche Erwägungen einer möglichen Substitution menschlicher Arbeit durch Maschinen in der Folge zur Prekarisierung dieser Arbeiten führen - und zwar wahrscheinlicher noch als die tatsächliche Realisierung dieser Möglichkeiten -, zeigt sich etwa bereits in den Bereichen der Versandlogistik bei Amazon (siehe Staab/Nachtwey 2016: 466) und der Altenpflege (siehe Hellige/Meilwes/Seidel 2018: 114-115).

Mit Reckwitz' Thesen zur kulturellen Auf- und Abwertung bestimmter Berufe und Tätigkeiten (siehe insbesondere S. 64-68) lassen sich also aktuelle Spannungsfelder im Bereich journalistischer Arbeit gesellschaftstheoretisch einordnen und erklären. Für die kommunikationswissenschaftliche Forschung in diesem Bereich provoziert diese Perspektive dabei die häufig vernachlässigte Berücksichtigung der gesellschaftlichen Bedingungen für das Verhältnis technologischer Entwicklungen und der Veränderung von Arbeitsbedingungen insbesondere im Niedriglohnsektor, die für eine kritische Auseinandersetzung etwa mit den Potenzialen sogenannter ,künstlicher Intelligenz' dringend notwendig ist.

\section{3 Öffentliche Kommunikation}

Schließlich lassen sich mit Reckwitz' Theorie der Spätmoderne Hypothesen zu möglichen Veränderungen der öffentlichen Kommunikation seit den 1970er Jahren (neu) formulieren. Zum Tragen kommen hier insbesondere die Annahmen einer „Affektintensivierung“ (S. 17) und Polarisierung der Gesellschaft. Sollten sie zutreffen, müssten sich in den vergangenen 50 Jahren etwa die Nachrichtenwertfaktoren - zumindest in bestimmten Ressorts und Formaten - merklich verschoben haben. Auch wenn Langzeitstudien hierzu nur schwer zu realisieren und daher recht selten sind (siehe Kepplinger 1998: 23-24), weisen einige Studien tatsächlich darauf hin, dass ein solcher Trend zu mehr Personalisierung, Konflikt, Aggression/ Gewalt und Emotionen besteht (siehe Ruhrmann/Göbbel 2007: 3-17). 
Die Vermutung einer Affektintensivierung der öffentlichen Kommunikation ist zwar nicht neu, sondern eine zentrale Annahme der öffentlichkeitstheoretischen Konzeptionen zu ,affective publics“ (siehe Lünenborg 2019); gleichwohl liefert Reckwitz hier einen möglichen gesellschaftstheoretischen Rahmen, mit dem sich die bisherige Theoriearbeit in diesem Bereich verknüpfen ließe. Zudem bieten die Thesen zum „Paternostereffekt“ der kulturellen Auf- und Abwertung verschiedener Lebensstile sowie zu der daraus erwachsenen Drei-Klassen-Gesellschaft einen neuen Ansatz zur Erklärung der verstärkten Dissonanz und Polarisierung öffentlicher Kommunikation (siehe Pfetsch et al. 2018). Phänomene wie der politische Populismus in Nordamerika und Europa (siehe Aalberg et al. 2017, Norris/Inglehart 2019, Koppetsch 2019) und der starke Widerstand gegen Liberalismus, Multikulturalismus, Gleichstellung und Ökologie (siehe Vertovec/Wessendorf 2010, Kuhar/Paternotte 2017, Garrard et al. 2019) ließen sich dann aus eben jenem Konflikt kultureller Klassen heraus erklären, der sich im Zuge der allmählichen Umstellung der sozialen Logiken und der Entfaltung der spätmodernen Gesellschaft über die Zeit aufgebaut und zunehmend verschärft hat.

Der maßgebliche Mechanismus dieser Polarisierung liegt dabei in der „Kulturalisierung der Ungleichheit“ (S. 350) zwischen den drei Klassen vor dem Hintergrund ihrer jeweiligen Lebensführung. Während sich die akademische Mittelklasse über die Singularisierung des eigenen Lebens von den beiden anderen Klassen abzuheben und zu singularisieren versucht, entgegnen diese wiederum mit der Kulturalisierung ihres Lebensstils, die mit einer expliziten Ablehnung des Lebensstils der akademischen Mittelklasse einhergeht. Verstärkt wird die daraus resultierende Polarisierung zudem durch einen „Aufstieg des Kulturessentialismus“, den Reckwitz an einer Zunahme kollektiver Identitätsbildung mit entsprechender Identitätspolitik, partikularer Neogemeinschaften, der Selbst- und Fremdkulturalisierung von Ethnien sowie einem Kulturnationalismus und religiösem Fundamentalismus festmacht (siehe dazu ausführlich Reckwitz 2019: 29-61).

Schließlich hält Reckwitz mit dem Konzept der „negativen Singularitäten“ (S. 426) eine Erklärung für die auf ein mediales Publikum ausgerichtete ,,demonstrative Gewalt“" (S. 424) von Terrorakten und Amokläufen bereit. Wie zielgenau sich die Inszenierung solcher Taten in die Logik einer Gesellschaft der Singularitäten einfügt, beschreibt Reckwitz dabei wie folgt:

„Nun ist es nicht das bewunderte, das interessante oder originelle Besondere, sondern das erschreckende und abstoßend Besondere, das den Blick auf sich zieht. Die spätmoderne Positivkultur der Affektivität wird damit durch einen Akt obszöner Grausamkeit durchbrochen. Es gilt: In einer Positivkultur der Affekte berührt nichts so stark wie übermäßige Negativität, das Publikum sieht sich nachgerade gezwungen, die abscheuliche Grandiosität der Tat zu zertifizieren." (S. 426) 
Neben den Spannungen und Konflikten, die sich aus der ausgrenzenden Kulturalisierung des jeweils eigenen Lebensstils und dem Aufstieg des Kulturessentialismus ergeben und die sich dann in der öffentlichen Kommunikation entladen, sei die öffentliche Inszenierung außerordentlicher Gewalt wie auch anderer Formen negativer Singularisierung (im wirkmächtigen Kontrast zur üblicherweise um positive Affizierung bemühte Praktiken) deshalb ein weiteres „Signum der Spätmoderne" (S. 423). Für eine von Reckwitz inspirierte Theoriearbeit im Bereich der Öffentlichkeitstheorie und entsprechender Theorien mittlerer Reichweite (etwa zu Skandalen, öffentlichen Streitfragen und Konflikten, Risiko- und Krisenkommunikation oder Wahlkampfkampagnen) wären dies also erste Ansatzpunkte.

\section{$4 \quad$ Fazit}

Dieser Beitrag hatte zum Ziel, Reckwitz' gesellschaftstheoretischen Entwurf einer spätmodernen Gesellschaft der Singularitäten auf sein öffentlichkeitstheoretisches Potenzial hin zu prüfen. Dazu wurden die Grundzüge der Theorie vorgestellt, die wichtigsten Kritikpunkte zusammengefasst und die maßgeblichen Thesen und Unterscheidungen der Theorie auf drei klassische Bereiche der Öffentlichkeitstheorie angewandt: die Konzeption von Öffentlichkeit, die Bedingungen journalistischer Arbeit sowie mögliche Konsequenzen für die öffentliche Kommunikation. Dabei wurde erstens deutlich, dass sich mit Hilfe der Theorie eine zweifache Strukturhaftigkeit von Öffentlichkeit modellieren lässt, die sich in Folge eines Strukturwandels hin zur Spätmoderne herausgebildet haben könnte. Unterscheiden lassen sich in diesem Zusammenhang zweitens ein ,Journalismus des Allgemeinen' und ein ,Journalismus des Besonderen', die sich in ihren sozialen Praktiken an unterschiedlichen Logiken ausrichten und sehr verschieden wertgeschätzt werden, was zu einer teilweisen Prekarisierung und zu Spannungen innerhalb des Journalismus führt. Drittens bietet die Theorie einer kulturellen Drei-Klassen-Gesellschaft eine gesellschaftstheoretische Erklärung von Affektintensivierung, zunehmender Dissonanz und Polarisierung öffentlicher Kommunikation.

Ein gesellschaftstheoretischer Zugriff auf Fragen zum digitalen Strukturwandel der Öffentlichkeit ist also in der Lage, theoretisch und empirisch anschlussfähige Erklärungsansätze zu liefern, die parallele Entwicklungen aus verschiedenen Gesellschaftsbereichen berücksichtigen und dadurch umfangreiche Erklärkraft erlangen. Wie beschrieben, haben es die Kommunikationswissenschaft und die Soziologie bei der Beschreibung und Erklärung gesellschaftlicher Strukturveränderungen dabei grundsätzlich mit der erkenntnistheoretischen und damit methodischen Herausforderung zu tun, eine sich wandelnde oder gar schon gewandelte Struktur von Gesellschaft und Öffentlichkeit aus einer begrifflichen, theoretischen 
und disziplinären Tradition heraus zu beschreiben, die an eben jener Gesellschaftsstruktur geschult und entwickelt wurde, die der These des Strukturwandels zufolge nun überholt sein soll. Zur Adressierung dieser Herausforderung wurde im vorliegenden Beitrag vorgeschlagen, soziologische Gesellschaftsdiagnosen im Sinne einer gesellschaftstheoretischen Exploration in die Theoriearbeit aktiv miteinzubeziehen und sich ihre Charakteristika der spezifischen Fokussierung und der spekulativ verallgemeinernden Projektion zunutze zu machen.

Dabei müssen allerdings auch die Grenzen eines solchen Vorgehens im Blick behalten werden. So ist davon auszugehen, dass sowohl die Fokussierung auf spezifische Aspekte als auch die Schwerpunktsetzung in der spekulativen Projektion anfällig sind für subjektive Verzerrungen und eine akademische „Klassenspezifik“ (Koppetsch). Eine zweite Schwierigkeit ergibt sich durch den Blick auf die „,ich abzeichnende Zukunft“ (Beck), da es sich dabei dem Prinzip nach um eine Analyse des noch Unbekannten und Unerschlossenen handelt. So liefert zunächst vor allem das Sichtbare erste Anhaltspunkte dafür, worin das Neue und Andere dieser Zukunft bestehen könnte. Deshalb ist auch die Kritik, Reckwitz verwechsele „die Selbstbeschreibung der spätmodernen Gesellschaft mit ihren Strukturen“ (Koppetsch), nicht überraschend, aber umso ernster zu nehmen; denn es besteht die Gefahr, dass sich die Theorie vor allem durch ihre Konsistenz mit dem Offensichtlichen plausibilisiert. Weiter verstärkt wird dies noch durch die Einführung neuer Begriffe - wie in diesem Fall dem der ,Singularitäten'. Solche neuen Begriffe sind nicht nur „unverbraucht“ und „unbelastet“ (S. 47), wie Reckwitz meint, sondern dadurch auch noch weitgehend unbestimmt und vage. Die Kritik an der Unschärfe des Singularitätskonzepts macht dies deutlich. Sobald noch vage Begriffe zum tragenden Element einer Theorie werden, kann es dazu kommen, dass ihre Diffusität eine Plausibilisierung und Anschlussfähigkeit der Theorie erlaubt, die sich bei näherer Begriffsbestimmung nicht mehr aufrechterhalten lässt.

Die Frage des Mediensymposiums nach der Rolle der Digitalisierung für den Strukturwandel der Öffentlichkeit lässt sich schließlich wie folgt beantworten: $\mathrm{Zu}$ nächst wäre mit Reckwitz 'Theorie der Annahme zuzustimmen, dass die Digitalisierung und insbesondere das Internet maßgebliche Treiber für den gesellschaftlichen Strukturwandel von der industriellen Moderne hin zur Spätmoderne darstellen. Gleichwohl kann die Umstellung der sozialen Logik vom Primat des Allgemeinen auf jenen des Besonderen bzw. Singulären, die Reckwitz zufolge diesem Strukturwandel zugrunde liegt, dieser Theorie nach nicht alleine durch die Digitalisierung erklärt werden. Relevant seien zudem ein sozio-kultureller Wandel seit den 1970er Jahren sowie die Umstellung der industriellen auf eine kulturkapitalistische Ökonomie. Diese drei Treiber des Strukturwandels hätten sich, so Reckwitz, überlagert und gegenseitig verstärkt. 
Das konkrete Verhältnis dieser drei Faktoren ist mit seinem Hinweis auf ihre „historische Koinzidenz“ allerdings noch recht unzureichend beschrieben. Denn auch wenn die technischen Grundlagen für Digitalisierung und des Internets bereits weitaus früher gelegt wurden, so erlangen sie doch erst ab den 1990er Jahren jene gesellschaftliche Relevanz, die für nachhaltige Auswirkungen auf die Gesellschaftsstruktur nötig wären (siehe Warnke 2011). Insofern ist mit Reckwitz nicht von einer digitalen Zäsur für die Beschaffenheit von Gesellschaft und Öffentlichkeit auszugehen als vielmehr von einem allmählichen Wandel, der durch Veränderungen in verschiedenen Gesellschaftsbereichen angetrieben und verstärkt wird. Für eine Analyse der Ursachen und des Verlaufs eines erneuten Strukturwandels von Gesellschaft und Öffentlichkeit bedarf es dementsprechend einer Konkretisierung des historischen Zusammenspiels dieser Veränderungen wie sie etwa in den Arbeiten zur „kalifornischen Ideologie“ (Barbrook/Cameron 1996, Diederichsen/ Franke 2013) bereits ansatzweise herausgearbeitet wurden.

\section{Literatur}

Aalberg, Toril/Esser, Frank/Reinemann, Carsten/Stromback, Jesper/De Vreese, Claes (Hg.) (2017): Populist Political Communication in Europe. New York, London: Routledge.

Bach, Clemens (2018): Andreas Reckwitz (2017): Die Gesellschaft der Singularitäten. Zum Strukturwandel der Moderne. Berlin: Suhrkamp Verlag. 480 Seiten. In: Vierteljahrsschrift für wissenschaftliche Pädagogik 94(1), S. 170-176.

Baecker, Dirk (2007): Studien zur nächsten Gesellschaft. Frankfurt am Main: Suhrkamp.

Baecker, Dirk (2018): 4.0 oder Die Lücke die der Rechner lässt. Berlin: Merve.

Barbrook, Richard/Cameron, Andy (1996): The Californian Ideology. In: Science as Culture 6(1), S. 44-72.

Beck, Ulrich (1986): Risikogesellschaft: Auf dem Weg in eine andere Moderne. Frankfurt am Main: Suhrkamp.

Blöbaum, Bernd (2000): Strukturwandel des Journalismus - Strukturwandel von Öffentlichkeit. In Otfried Jarren/Kurt Imhof/Roger Blum (Hg.): Zerfall der Öffentlichkeit? S. 135-147. Wiesbaden: VS.

Bogner, Alexander (2018): Gesellschaftsdiagnosen. Ein Überblick (3. Aufl.). Weinheim, Basel: Beltz Juventa.

Boltanski, Luc/Chiapello, Ève (2003): Der neue Geist des Kapitalismus. Konstanz: UVK.

Bourdieu, Pierre (1972): Entwurf einer Theorie der Praxis (auf der ethnologischen Grundlage der kabylischen Gesellschaft). Frankfurt am Main: Suhrkamp.

Castells, Manuel (2001): The Internet Galaxy. Reflections on Internet, Business, and Society. Oxford: Oxford University Press.

Clerwall, Christer (2014): Enter the Robot Journalist. Users' perceptions of automated content. In: Journalism Practice 8(5), S. 519-531.

Diederichsen, Diedrich/Franke, Anselm (2013): The Whole Earth: Kalifornien und das Verschwinden des Außen. Berlin: Sternberg Press. 
Dimbath, Oliver (2016): Soziologische Zeitdiagnostik. Konstanz, München: UTB.

Dörr, Konstantin Nicholas (2015): Mapping the field of Algorithmic Journalism. In: Digital Journalism 4(6), S. 700-722.

Donges, Patrick/Jarren, Otfried (1998): Öffentlichkeit und Öffentliche Meinung. In Heinz Bonfadelli/Walter Hättenschwiler (Hg.): Einführung in die Publizistikwissenschaft. Eine Textsammlung, S. 95-110. Zürich: IPMZ.

Eberlein, Undine (2000): Einzigartigkeit: Das romantische Individualitätskonzept der Moderne. Frankfurt am Main: Campus.

Eisenegger, Mark (2017): Vorwort: Digitaler Strukturwandel der Öffentlichkeit - professionelle Informationsmedien nötiger denn je! In fög (Hg.): Qualität der Medien. Schweiz - Suisse - Svizzera. Jahrbuch 2017, S. 7-16. Basel: Schwabe.

Frank, Daniel (2018): Spätmoderne oder die Explosion des Besonderen. In: Zeitschrift für Technikfolgenabschätzung in Theorie und Praxis 27(2), S. 72-73.

Fraser, Nancy (1990): Rethinking the Public Sphere: A Contribution to the Critique of Actually Existing Democracy. In: Social Text 25/26, S. 56-80.

Fürst, Silke (2013): Öffentlichkeitsresonanz als Nachrichtenfaktor - Zum Wandel der Nachrichtenselektion. In: Medien Journal 37(2), S. 4-15.

Garrard, Greg/Goodbody, Axel/Handley, George B./Posthumus, Stephanie (2019): Climate Change Scepticism: A Transnational Ecocritical Analysis. London: Bloomsbury.

Gerhards, Jürgen (2001): Der Aufstand des Publikums. Eine systemtheoretische Interpretation des Kulturwandels in Deutschland zwischen 1960 und 1989. In: Zeitschrift für Soziologie 30(3), S. 163-184.

Gerhards, Jürgen/Neidhardt, Friedhelm (1990): Strukturen und Funktionen moderner Öffentlichkeit: Fragestellungen und Ansätze, WZB Discussion Paper, No. FS III 90-101. Berlin: Wissenschaftszentrum Berlin für Sozialforschung.

Giddens, Anthony (1984): Die Konstitution der Gesellschaft. Grundzüge einer Theorie der Strukturierung. Frankfurt am Main: Campus.

Graefe, Andreas (2016): Guide to Automated Journalism [Report]. New York: Tow Center for Digital Journalism. Abgerufen von https://towcenter.gitbooks.io/guide-to-automated-journalism/content/

Gümüsay, Ali Aslan (2018): Book Review: Andreas Reckwitz Die Gesellschaft der Singularitäten - Zum Strukturwandel der Moderne [The society of singularities - On the transformation of modernity]. In: Organization Studies 39(10), S. 1492-1495.

Habermas, Jürgen (1962): Strukturwandel der Öffentlichkeit. Untersuchungen zu einer Kategorie der bürgerlichen Gesellschaft. Frankfurt am Main: Suhrkamp.

Haim, Mario (2019): Die Orientierung von Online-Journalismus an seinen Publika: Anforderung, Antizipation, Anspruch. Wiesbaden: Springer VS.

Hasebrink, Uwe (2019): Strukturwandel von Öffentlichkeit: Wie tragen Individuen durch ihre Mediennutzung zum Strukturwandel von Öffentlichkeit bei? In Mark Eisenegger/ Linards Udris/Patrik Ettinger (Hg.): Wandel der Öffentlichkeit und der Gesellschaft Gedenkschrift für Kurt Imhof, S. 407-417. Wiesbaden: Springer.

Hellige, Barbara/Meilwes, Michael/Seidel, Sabine (2018): Digitalisierung und Sorgeverhältnisse - ein unauflöslicher Widerspruch? In Mario A. Pfannstiel/Sandra Krammer/ Walter Swoboda (Hg.): Digitale Transformation von Dienstleistungen im Gesundheitswesen IV: Impulse für die Pflegeorganisation, S. 113-133. Wiesbaden: Springer. 
Hohnsträter, Dirk (2018): Kultur, Konsum und die Gesellschaft der Singularitäten. Soziopolis. Abgerufen von https://soziopolis.de/beobachten/kultur/artikel/reckwitz-buchforum-7-die-gesellschaft-der-singularitaeten/

Honneth, Axel (1994): Desintegration: Bruchstücke einer soziologischen Zeitdiagnose. Frankfurt am Main: Fischer.

Horkheimer, Max/Adorno, Theodor W. (1944[2003]): Kulturindustrie. Aufklärung als Massenbetrug. In dies. (Hg.): Dialektik der Aufklärung. Philosophische Fragmente, S. 128176. Frankfurt am Main: Fischer.

Imhof, Kurt (2003a): Öffentlichkeitstheorien. In Günter Bentele/Hans-Bernd Brosius/Otfried Jarren (Hg.): Öffentliche Kommunikation: Handbuch Kommunikations- und Medienwissenschaft, S. 193-209. Wiesbaden: VS.

Imhof, Kurt (2003b): Wandel der Gesellschaft im Licht öffentlicher Kommunikation. In Markus Behmer/Friedrich Krotz/Rudolf Stöber/Carsten Winter (Hg.): Medienentwicklung und gesellschaftlicher Wandel. Beiträge zu einer theoretischen und empirischen Herausforderung und Geschichte, S. 153-182. Wiesbaden: Westdeutscher Verlag.

Imhof, Kurt (2008): Theorie der Öffentlichkeit als Theorie der Moderne. In Carsten Winter/Andreas Hepp/Friedrich Krotz (Hg.): Theorien der Kommunikations- und Medienwissenschaft: Grundlegende Diskussionen, Forschungsfelder und Theorieentwicklungen, S. 65-89. Wiesbaden: VS.

Imhof, Kurt (2011): Die Krise der Öffentlichkeit: Kommunikation und Medien als Faktoren des sozialen Wandels. Frankfurt am Main: Campus.

Jarren, Otfried (2008): Massenmedien als Intermediäre: Zur anhaltenden Relevanz der Massenmedien für die öffentliche Kommunikation. Medien \& Kommunikationswissenschaft 56(3-4), 329-346.

Jarren, Otfried (2015): Ordnung durch Medien? In Klaus-Dieter Altmeppen/Patrick Donges/Matthias Künzler/Manuel Puppis/Ulrike Röttger/Hartmut Wessler (Hg.): Soziale Ordnung durch Kommunikation?, S. 27-50. Baden-Baden: Nomos.

Junge, Matthias (2016): Zeitdiagnose als Chance der Soziologie. In ders. (Hg.): Metaphern soziologischer Zeitdiagnosen, S. 51-60. Wiesbaden: Springer.

Kepplinger, Hans Mathias (1998): Der Nachrichtenwert der Nachrichtenfaktoren. In Christina Holtz-Bacha/Helmut Scherer/Norbert Waldmann (Hg.): Wie die Medien die Welt erschaffen und wie die Menschen darin leben, S. 19-38. Wiesbaden: VS.

Kieserling, André (2002): Evergreens der Sozialkunde. In: Frankfurter Allgemeine Sonntagszeitung vom 21.04.2002, S. 68.

Kilian, Thomas (2018): Gesellschafstheorie. In: Kölner Zeitschrift für Soziologie und Sozialpsychologie 70(2), S. 311-313.

Knöbl, Wolfgang (2017): Eine neue Moderne? Soziopolis. Angerufen von https://soziopolis.de/beobachten/kultur/artikel/reckwitz-buchforum-1-die-gesellschaft-der-singularitaeten/

Köhler, Volker (2019): Moderne zwischen Allgemeinem und Besonderem. In: Neue Politische Literatur 64(1), S. 109-112.

Kohring, Matthias (2006): Öffentlichkeit als Funktionssystem der modernen Gesellschaft. Zur Motivationskraft der Mehrsystemzugehörigkeit. In Andreas Ziemann (Hg.): $\mathrm{Me}$ dien der Gesellschaft - Gesellschaft der Medien, S. 161-182. Konstanz: UVK. 
Koppetsch, Cornelia (2017): Eine kultursoziologische Kartierung der Gegenwart - doch stimmt sie auch? Soziopolis. https://soziopolis.de/beobachten/kultur/artikel/reckwitzbuchforum-2-die-gesellschaft-der-singularitaeten/

Koppetsch, Cornelia (2019): Die Gesellschaft des Zorns: Rechtspopulismus im globalen Zeitalter. Bielefeld: transkript.

Kramp, Leif (2013): Profession am Scheideweg: Journalismus zwischen Aufbruch und Existenzängsten. In Leif Kramp/Leonard Novy/Dennis Ballwieser/Karsten Wenzlaff (Hg.): Journalismus in der digitalen Moderne: Einsichten - Ansichten - Aussichten, S. 33-62. Wiesbaden: Springer.

Krüger, Uwe (2016): Mainstream: Warum wir den Medien nicht mehr trauen. München: C.H. Beck.

Kuhar, Roman/Paternotte, David (Hg.) (2017): Anti-Gender Campaigns in Europe: Mobilizing Against Equality. London, New York: Rowman \& Littlefield International.

Lichtblau, Klaus (1991): Soziologie und Zeitdiagnose. Oder: Die Moderne im Selbstbezug. In Stefan Müller-Doohm (Hg.): Jenseits der Utopie. Theoriekritik der Gegenwart, S. 15-47. Frankfurt am Main: Suhrkamp.

Lichtblau, Klaus (1998): Die Selbstunterscheidungen der Moderne. In Johannes Weiß (Hg.): Mehrdeutigkeiten der Moderne, S. 43-87. Kassel: Kassel University Press.

Löw, Martina (2019): Die Singularität der sozialen Logik in der Spätmoderne? Über Transformation, Konflikt und Raum in Andreas Reckwitz' Buch „Die Gesellschaft der Singularitäten“. In: Soziologische Revue 42(1), S. 3-10.

Loosen, Wiebke/Schmidt, Jan-Hinrik (2012): (Re-)Discovering the audience: The relationship between journalism and audience in networked digital media. Information, Communication \& Society 15(6), 867-887.

Lünenborg, Margreth (2019): Affective publics. In Jan Slaby/Christian von Scheve (Hg.): Affective Societies: Key Concepts, S. 319-329. London: Routledge.

Luhmann, Niklas (1984): Soziale Systeme. Grundriß einer allgemeinen Theorie. Frankfurt am Main: Suhrkamp.

Luhmann, Niklas (1987): Tautologie und Paradoxie in den Selbstbeschreibungen der modernen Gesellschaft. In: Zeitschrift für Soziologie 16(3), S. 161-174.

Marcinkowski, Frank (1993): Publizistik als autopoietisches System. Politik und Massenmedien. Eine systemtheoretische Analyse. Opladen: Westdeutscher Verlag.

Meyen, Michael (2018): Singularisierung vs. Medialisierung. Medienrealität. Abgerufen von https://medienblog.hypotheses.org/1384

Moebius, Stephan (2018): Die Sakralität des Singulären und ihre symbolische Macht. Soziopolis. Abgerufen von https://soziopolis.de/beobachten/kultur/artikel/reckwitzbuchforum-9-die-gesellschaft-der-singularitaeten/

Müller, Philipp (2018): Polarisierung des Publikums. Wie sich die Beziehung zwischen Journalismus und Bürgern verändert - und warum. In Peter Limbourg/Ronald Grätz (Hg.): Meinungsmache im Netz: Fake News, Bots und Hate Speech, S. 33-43. Göttingen: Steidl.

Neuberger, Christoph (2002): Alles Content, oder was? Vom Unsichtbarwerden des Journalismus im Internet. In Ralf Hohlfeld/Klaus Meier/Christoph Neuberger (Hg.): Innovationen im Journalismus: Forschung für die Praxis, S. 25-69. Münster: LIT. 
Neuberger, Christoph (2014): Konflikt, Konkurrenz und Kooperation. Interaktionsmodi in einer Theorie der dynamischen Netzwerköffentlichkeit. In: Medien \& Kommunikationswissenschaft 62(4), S. 567-587.

Norris, Pippa/Inglehart, Ronald (2019): Cultural Backlash: Trump, Brexit, and Authoritarian Populism. Cambridge: Cambridge University Press.

Nuernbergk, Christian (2013): Anschlusskommunikation in der Netzwerköffentlichkeit. Ein inhalts- und netzwerkanalytischer Vergleich der Kommunikation im "Social Web“ zum G8-Gipfel von Heiligendamm. Baden-Baden: Nomos.

Osrecki, Fran (2011): Die Diagnosegesellschaft: Zeitdiagnostik zwischen Soziologie und medialer Popularität. Bielefeld: transkript.

Papacharissi, Zizi (2015): Affective publics: sentiment, technology, and politics. Oxford: Oxford University Press.

Parsons, Talcott (1971): The System of Modern Societies. Englewood Cliffs: Prentice-Hall.

Pfetsch, Barbara/Löblich, Maria/Eilders, Christiane (2018): Dissonante Öffentlichkeiten als Perspektive kommunikationswissenschaftlicher Theoriebildung. In: Publizistik 63(4), S. 477-495.

Reckwitz, Andreas (2003): Grundelemente einer Theorie sozialer Praktiken. Eine sozialtheoretische Perspektive. In: Zeitschrift für Soziologie 31(4), S. 282-301.

Reckwitz, Andreas (2017a): Die Gesellschaft der Singularitäten. Zum Strukturwandel der Moderne. Berlin: Suhrkamp.

Reckwitz, Andreas (2017b): Eine Replik. Soziopolis. Abgerufen von https://soziopolis.de/beobachten/kultur/artikel/reckwitz-buchforum-4-die-gesellschaft-der-singularitaeten/

Reckwitz, Andreas (2018): Einige nicht-abschließende Bemerkungen. Soziopolis. Abgerufen von https://soziopolis.de/beobachten/kultur/artikel/reckwitz-buchforum-10-diegesellschaft-der-singularitaeten/

Reckwitz, Andreas (2019): Das Ende der Illusionen: Politik, Ökonomie und Kultur in der Spätmoderne. Berlin: Suhrkamp.

Reese-Schäfer, Walter (1996): Zeitdiagnose als wissenschaftliche Aufgabe. Berliner Journal für Soziologie 6(3), 377-390.

Reitz, Tilman (2019): Späte Zeitdiagnose und soziologische Systematisierung. In: Soziologische Revue 42(1), S. 11-19.

Rosa, Hartmut (2018): Dynamische Stabilisierung oder metrische Singularisierung? Soziopolis. Abgerufen von https://soziopolis.de/beobachten/kultur/artikel/reckwitz-buchforum-8-die-gesellschaft-der-singularitaeten/

Rühl, Manfred (1980): Journalismus und Gesellschaft. Bestandsaufnahme und Theorieentwurf. Mainz.

Ruhrmann, Georg/Göbbel, Roland (2007): Veränderung der Nachrichtenfaktoren und Auswirkungen auf die journalistische Praxis in Deutschland. Abschlussbericht für netzwerk recherche e.V. Abgerufen von https://netzwerkrecherche.org/files/nr-studienachrichtenfaktoren.pdf

Saar, Martin (2018): Affekt und Singularität. Soziopolis. Abgerufen von https://soziopolis.de/beobachten/kultur/artikel/reckwitz-buchforum-6-die-gesellschaft-der-singularitaeten/ 
Schatto-Eckrodt, Tim/Boberg, Svenja/Wintterlin, Florian/Frischlich, Lena/Quandt, Thorsten (2019): Bedrohte Deliberation. Information Warfare und Desinformation als Bedrohung digitaler Öffentlichkeiten. In: Communicatio Socialis 52(2), S. 147-158.

Schatzki, Theodore R. (1996): Social Practices: A Wittgensteinian approach to human activity and the social. Cambridge: Cambridge University Press.

Schindler, Johanna/Fortkord, Claudia/Posthumus, Lone/Obermaier, Magdalena/Reinemann, Carsten/Fawzi, Nayla (2018): Woher kommt und wozu führt Medienfeindlichkeit? Zum Zusammenhang von populistischen Einstellungen, Medienfeindlichkeit, negativen Emotionen und Partizipation. In: Medien \& Kommunikationswissenschaft 66(3), 283-301.

Schmidt, Jan-Hinrik/Merten, Lisa/Hasebrink, Uwe/Petrich, Isabelle/Rolfs, Amelie (2017): Zur Relevanz von Online-Intermediären für die Meinungsbildung. Hamburg: Verlag Hans-Bredow-Institut (Arbeitspapiere des Hans-Bredow-Instituts Nr. 40).

Schnabel, Annette (2019): Singularitäten soziologisieren. In: Soziologische Revue 42(1), S. 20-26.

Simmel, Georg (1901[1995]): Die beiden Formen des Individualismus. In Rüdiger Kramme/Angela Rammstedt/Otthein Rammstedt (Hg.): Georg Simmel - Aufsätze und Abhandlungen 1901-1908, Band 1, S. 49-56. Suhrkamp: Frankfurt am Main.

Srnicek, Nick (2016): Platform Capitalism. Cambridge: Polity Press.

Staab, Philipp/Nachtwey, Oliver (2016): Market and Labour Control in Digital Capitalism. In: tripleC 14(2), 457-474.

Star, Susan Leigh (1999): The Ethnography of Infrastructure. In: American Behavioral Scientist 43(3), S. 377-391.

Stark, Birgit/Magin, Melanie (2019): Neuer Strukturwandel der Öffentlichkeit durch Informationsintermediäre: Wie Facebook, Google \& Co. die Medien und den Journalismus verändern. In: Mark Eisenegger/Linards Udris/Patrik Ettinger (Hg.): Wandel der Öffentlichkeit und der Gesellschaft: Gedenkschrift für Kurt Imhof, S. 377-406. Wiesbaden: Springer.

Trautmann, Felix (2017): Die demokratische Gesellschaft ist keine Gesellschaft von Singularitäten. Soziopolis. Abgerufen von https://soziopolis.de/beobachten/kultur/arti$\mathrm{kel} /$ reckwitz-buchforum-5-die-gesellschaft-der-singularitaeten/

van Dijck, José/Poell, Thomas/de Waal, Martijn (2018): The Platform Society: Public Values in a Connective World. Oxford: Oxford University Press.

Vertovec, Steven/Wessendorf, Susanne (Hg.) (2010): The Multiculturalism Backlash: European discourses, policies and practices. New York, London: Routledge.

Vogel, Berthold (2017): Mehr Nostalgie wagen!? Soziopolis. Abgerufen von https://soziopolis.de/beobachten/kultur/artikel/reckwitz-buchforum-3-die-gesellschaft-der-singularitaeten/

Wagner, Peter (2019): Singularity: A New Key for the Sociological Diagnosis of the Present Time? - Andreas Reckwitz, Die Gesellschaft der Singularitäten. Zum Strukturwandel der Moderne (Berlin, Suhrkamp Verlag, 2017). In: European Journal of Sociology 59(3), S. 524-532.

Warnke, Martin (2011): Theorien des Internet zur Einführung. Hamburg: Junius. 